\title{
Las baterías como residuos tecnológicos contaminantes: Un reto de la educación ambiental
}

\author{
Batteries as technological contaminants waste: \\ a challenge for environmental education
}

\author{
Nancy Rocío Espinosa Escobar \\ Magíster en Educación Ambiental \\ Investigadora independiente \\ Bucaramanga, Santander, Colombia \\ Correo electrónico: rocio.e5@hotmail.com
}

Recibido: 30 de mayo de 2018

Aceptado: 23 de julio de 2018

\begin{abstract}
Cómo citar este artículo:
Espinosa, N.R. (2019). Las baterías como residuos tecnológicos contaminantes: Un reto de la educación ambiental Espiral, Revista de Docencia e Investigación, 9(1), 71 - 85.
\end{abstract}

\section{Resumen}

Este artículo de investigación presenta el desarrollo de una estrategia de educación ambiental, orientada al manejo responsable de residuos tecnológicos más frecuentes en dos instituciones educativas del departamento de Boyacá. Esta investigación se desarrolla bajo un enfoque preventivo alrededor de la problemática que surge en torno al mercado de equipos tecnológicos, como portátiles, tabletas y celulares en Colombia, que ha crecido rápidamente, con la subsiguiente producción en cantidades de baterías desechadas, que contienen elevadas concentraciones de metales, como oro, plata, níquel, cadmio, mercurio, entre otros; los cuales al disponerlos de manera inadecuada afectan el agua, el aire y el suelo. Sumado a lo anterior, en el mercado colombiano no se evidencian unos controles claros para la disposición de los residuos tecnológicos, lo que hace necesario desarrollar una estrategia de educación ambiental para la disposición de baterías de equipos tecnológicos, como tabletas, portátiles y teléfonos celulares en dos instituciones del departamento de Boyacá, que se fundamenta en la investigación - acción bajo un enfoque mixto. Se utiliza como técnicas de recolección de la información la encuesta, los grupos de enfoque y la entrevista semiestructurada. Por medio de estos grupos se desarrollan talleres investigativos para fortalecer la implementación de estrategias en educación ambiental con respecto a la disposición de las baterías de equipos tecnológicos, con el fin de promover conocimientos, actitudes y hábitos que minimicen el impacto ambiental que genera la basura tecnológica.

Palabras claves: Estrategia, educación ambiental, baterías, equipos tecnológicos, residuos tecnológicos.

\section{Abstract}

This research article presents the development of an Environmental Education Strategy oriented to the responsible management of the most frequent technological waste in two Educational Institutions of the department of Boyacá. This research is developed under a preventive approach around the problems that arise around the market of technological equipment such as laptops, tablets and cell phones in Colombia that has grown rapidly, with the subsequent production in quantities of discarded batteries, which contain high concentrations of metals such as gold, silver, nickel, cadmium, mercury among others; which, when disposed of improperly, affect resources such as water, air and soil. In addition to the above, the Colombian market does not showclear controls for the disposal of technological waste, which makes it necessary to develop an Environmental Education Strategy for the disposal of batteries of technological equipment such as tablets, laptops and cell phones in two. Institutions of the department of Boyacá, which is based on action research under a mixed approach. The survey, the focus groups and the semistructured interview are used as information gathering techniques. Through these groups research workshops are developed to strengthen the implementation of Strategies in Environmental Education with respect to the disposition of the batteries of technological equipment, in order to promote knowledge, attitudes and habits that minimize the environmental impact generated by technological waste.

Keywords: Strategy, environmental education, batteries, technological equipment, technological waste. 


\section{Introducción}

En Colombia se han llevado a cabo importantes esfuerzos legislativos alrededor de la educación ambiental, reflejados en el Código Nacional de los Recursos Naturales Renovables y de Protección del Medio Ambiente, el cual presenta limitaciones por cuanto insiste solamente en la implementación de la educación ambiental por medio de cursos de ecología, de preservación de los recursos naturales y proyección de jornadas ecológicas en las instituciones educativas.

Bajo el marco de la educación ambiental y como resultado de la reflexión nacida en diferentes momentos del desarrollo de la Maestría en Educación Ambiental, surge la inquietud en relación con el comportamiento de las comunidades educativas frente a la proliferación de dispositivos tecnológicos, así como las prácticas relacionadas con la disposición de los residuos que estos generan, particularmente de las baterías de los equipos más utilizados. Se observa que los estudiantes tienden a depositar las baterías de los equipos tecnológicos en la basura o las almacenan en el hogar, debido a la falta de puntos de recolección; a esto se suma la carencia de acciones educativas que favorezcan el conocimiento acerca de las sustancias que las componen y los efectos que provocan en el medio ambiente; así como los beneficios del reciclaje tecnológico y los sitios certificados que se encargan de su tratamiento. Pareciera que sigue siendo costumbre, que al no experimentar de manera directa las consecuencias del manejo inadecuado de los recursos, se opte por una educación reactiva en lugar de procurar el desarrollo de una cultura preventiva.

De manera que se desarrolla una estrategia de educación ambiental que es entendida, según Mora, Quitiaquez y Rengifo $(2012$, p. 5) como un saber-hacer, un saber en acción, que reflexiona sobre la relación docente, estudiante y participante; así como entre la enseñanza y el aprendizaje; esta mirada conlleva la promoción de valores y aclaración de conceptos centrados en el fomento de actitudes, destrezas, habilidades y aptitudes necesarias para comprender y apreciar las interrelaciones entre el ser humano, su cultura y la naturaleza.

El proyecto de intervención se realiza en varias fases que van desde el diagnóstico sobre la realidad, luego el diseño, aplicación, evaluación y divulgación de la estrategia de educación ambiental orientada a la promoción de conocimiento sobre la adecuada disposición de baterías de equipos tecnológicos más utilizados, sus efectos en el medio ambiente y la importancia del desarrollo de hábitos y buenas prácticas para la preservación del medio ambiente.

\section{Metodología}

La presente investigación que tiene como propósito promover un proceso de educación ambiental para lograr una disposición adecuada de las baterías de equipos tecnológicos, se desarrolla desde un enfoque mixto, con énfasis en la investigación acción e involucra estudiantes de servicio social de dos instituciones educativas del departamento de Boyacá. Para el cumplimiento de sus objetivos se utiliza como técnicas e instrumentos: la encuesta para establecer el diagnóstico, el grupo focal para el desarrollo de talleres que constituyen la estrategia y la entrevista que tiene como fin la evaluación de la presente estrategia.

A partir de los resultados obtenidos se establecen tres categorías de análisis: Conocimiento, Actitud y Hábito que orientan los aspectos esenciales que se deben tener en cuenta en la promoción de una educación para la disposición adecuada de las baterías de equipos tecnológicos. Conforme a lo anterior, el conocimiento permite contemplar una realidad ambiental, asimilar y crear soluciones 
que están sujetas a una reinterpretación continua, y mediante un conocimiento pleno acerca de la necesidad de realizar una adecuada disposición de las baterías de equipos tecnológicos, se afronta el cambio de comportamiento y actitud. Así, a partir de los conocimientos previos de los estudiantes se encuentran los cimientos necesarios para la construcción de un conocimiento reflexivo y crítico de la problemática ambiental, con el ánimo de corregir hábitos mediante un acercamiento con la realidad, donde se genera en el estudiante, actitudes de valoración y respeto por el ambiente.

Además, se promueve el desarrollo de actitudes entendidas como estados de disposición mental que tienen un papel motivacional de orientación hacia la acción, que permite influir en la percepción y el pensamiento para el reconocimiento de la importancia y necesidad de realizar una adecuada disposición de las baterías, con el propósito de adquirir una responsabilidad tecnológica que afronta las consecuencias que ya son latentes para la preservación de los recursos que aún se conservan. Conjuntamente, la estrategia fortalece la adopción de hábitos que se refiere a los comportamientos de las personas, los cuales provocan un incremento de la gravedad del problema ambiental. Por tal motivo, es importante mencionar el impacto que genera la disposición de baterías en rellenos sanitarios, su incineración o acumulación en los hogares, las cuales son acciones que hace necesario un cambio de conducta, teniendo en cuenta la relación que mantiene el estudiante con los equipos tecnológicos, que requiere la construcción de hábitos enfocados a la disposición y uso racional de los residuos que genera la tecnología.

De manera que, a través de la metodología planteada se pretende contribuir con una propuesta de solución al problema ambiental que surge a partir de la basura tecnológica que afecta el bienestar de los seres vivos y el medio ambiente en general.

\section{Población y muestra}

La población que interviene en esta investigación la conforman integrantes de dos establecimientos educativos de carácter público, localizados en el departamento de Boyacá: El colegio de Boyacá de carácter urbano ubicado en la ciudad de Tunja y la Institución Educativa Panamericano Puente de Boyacá que pertenece al área urbana y se encuentra en el municipio de Ventaquemada. Son instituciones interesadas en la investigación ambiental, aspecto sustentado en los principios orientadores del PEI para la construcción de estrategias didácticas conducentes al cuidado y conservación del medio ambiente.

La muestra se elige por conveniencia, de cada institución participan sesenta estudiantes de séptimo a undécimo grado en el diagnóstico y para el diseño y aplicación de la estrategia veinticinco estudiantes de grado once, quienes adelantan el servicio social, y sesenta y cuatro estudiantes de grado noveno, distribuidos en las dos instituciones educativas, como se registra en la tabla 1.

Tabla 1. Descripción de las instituciones educativas del departamento de Boyacá

\begin{tabular}{|c|c|c|}
\hline & $\begin{array}{l}\text { Panamericano } \\
\text { Puente de Boyacá }\end{array}$ & $\begin{array}{c}\text { Colegio de } \\
\text { Boyacá }\end{array}$ \\
\hline Población & 650 & 4500 \\
\hline $\begin{array}{l}\text { Muestra } \\
\text { encuestada }\end{array}$ & 60 & 60 \\
\hline Grupo focal & 8 & 17 \\
\hline $\begin{array}{l}\text { Aplicación de } \\
\text { talleres }\end{array}$ & 30 & 34 \\
\hline
\end{tabular}

Fuente: Elaboración de la autora. 


\section{Resultados}

\section{Diagnóstico}

Para el desarrollo de la Estrategia en Educación Ambiental, como se mencionó, se inició con la aplicación de una encuesta de veinte preguntas cerradas a doce estudiantes de cada uno de los grados (séptimo a once), de dos instituciones educativas del departamento de Boyacá: Colegio de Boyacá y la Institución
Educativa Panamericano Puente de Boyacá. De manera que se aplicó a sesenta estudiantes por institución, con el propósito de identificar sus hábitos, actitudes y conocimientos.

La interpretación de las respuestas dadas a las preguntas formuladas en el diagnóstico permite establecer tres categorías de análisis: Conocimiento, Actitud y Hábito (tabla 2); estas orientan el diseño de la estrategia a partir de la observación, el pensamiento y la acción.

\section{Tabla 2. Categorías de análisis establecidas}

en el diagnóstico

\section{Categoría}

Subcategoría

Equipos tecnológicos

Conocimiento

Reglamentación

Responsabilidad tecnológica

Actitud

Indiferencia a la contaminación tecnológica

Adecuada disposición de residuos

Hábito

\section{Situación}

Desconocimiento acerca de los RAEE, composición de las baterías, disposición adecuada y centros de recolección adecuados.

Desconocimiento sobre Responsabilidad Extendida del Productor, y la ley de los RAEE.

Se identifica que la incineración y disposición en rellenos sanitarios, no es la opción adecuada para el manejo de las baterías al final de su ciclo de vida. Interés en el conocimiento de la disposición adecuada que deben recibir las baterías, por lo cual se desea participara en la estrategia de Educación Ambiental.

Desinterés por los efectos que puede generar la inadecuada disposición de las baterías.

Muy pocos estudiantes conocen los centros de acopio y hacen uso de estos.

Las baterías al final de su vida útil se almacenan en las residencias, o se arrojan a la basura.

Fuente: Elaboración de la autora. 


\section{Estrategia de Educación Ambiental}

De acuerdo con los resultados obtenidos en el diagnóstico, se identifica la necesidad de desarrollar una estrategia, que permite la generación de conocimientos, hábitos y actitudes en torno a una adecuada disposición de las baterías, ya que existe desconocimiento en cuanto a su composición, reglamentación, manejo adecuado, efectos en el medio ambiente, centros de acopio certificados para su disposición, consecuencias y responsabilidad frente al avance tecnológico.

Esta estrategia está planeada con base en el desarrollo de talleres que permiten la reflexión y el compromiso que se debe asumir frente a los residuos tecnológicos para cubrir las necesidades del contexto. De manera que la estrategia se acoge bajo el séptimo saber planteado por Morín, necesario para la educación del futuro, que se refiere a la formación de seres éticos y ciudadanos activos con conciencia política hacia la tierra y las demás personas (Avendaño, 2013, p. 3). Además, se orienta a través de la teoría de la Modificabilidad Estructural Cognitiva (MEC), dado su enfoque integrador e inclusivo, privilegiando la mediación acorde con las necesidades y desde el marco de unos criterios que permiten al sujeto aprender y modificarse si lo desea.

A partir de la teoría de la Modificabilidad Estructural Cognitiva (MEC) que ilumina la Estrategia de Educación Ambiental, se tiene en cuenta el modelo pedagógico autoestructurante relacionado con la Experiencia de Aprendizaje Mediado (EAM) planteado por Zubiria, que se define como el dispositivo para la ver- dadera modificabilidad del sujeto, donde es preciso atender ciertas características para lograr la interacción, como la intencionalidad y reciprocidad, la mediación del significado y la transcendencia (Avendaño, 2013, p. 6).

De acuerdo con el modelo pedagógico autoestructurante, los componentes que hacen parte del taller: propósito, introducción, metodología y retroalimentación, se orientan en primer lugar bajo el propósito de una educación que permite la construcción de actitudes y hábitos desde el interior de cada estudiante. Así mismo, el saber, como criterio clave de este modelo pedagógico, permite a partir de los conceptos claves y la metodología que integran cada taller, la promoción de estrategias por descubrimiento e invención, centrado en la dinámica e interés del estudiante, teniendo en cuenta que la metodología que guía el taller se combina en actividades, tales como trabajo individual y grupal, para el análisis y planteamiento de soluciones. Ofrece ventajas como el desarrollo del juicio y la habilidad mental para comprender procesos, determinar causas y escoger soluciones prácticas. Estimula el trabajo cooperativo, ejercita la actividad creadora y la iniciativa, favorece la participación y propicia que se comparta y aplique lo aprendido.

Por su misma naturaleza, el taller permite la aplicación de los conocimientos adquiridos en la disposición de las baterías como residuos tecnológicos con el ánimo de prevenir su impacto ambiental, debido a que este se plantea para la vivencia, la reflexión, la conceptualización y la acción; como síntesis del pensar, el sentir y el hacer bajo una actividad constructiva del participante. 
De modo que el taller ha sido elegido como el medio para el abordaje integral de situaciones y su transformación, mediante planes de trabajo que derivan del resultado arrojado en el diagnóstico y comprometen a diferentes actores de las instituciones educativas; de manera directa a los estudiantes de grado once, que adelantan el servicio social, quienes a su vez replican cada taller con sus compañeros de grado noveno. En la tabla 3 se registran los cuatro talleres, con su respectivo propósito, los conceptos clave, la metodología; que orienta el fortalecimiento de conceptos para la construcción de actitudes y hábitos, así como los resultados de su evaluación.

\section{Tabla 3. Descripción de los talleres de Educación Ambiental}

\begin{tabular}{|c|c|c|}
\hline Taller & \multicolumn{2}{|c|}{$\begin{array}{l}\text { 1. Desarrollo tecnológico, generación y } \\
\text { disposición de la basura electrónica }\end{array}$} \\
\hline Propósito & \multicolumn{2}{|c|}{$\begin{array}{l}\text { Reconocer los riesgos que representa la } \\
\text { inadecuada disposición de los residuos } \\
\text { tecnológicos, entre los cuales se destacan las } \\
\text { baterías }\end{array}$} \\
\hline Conceptos clave & & $\begin{array}{l}\text { RAEE } \\
\text { Baterías } \\
\text { Metales } \\
\text { Plásticos }\end{array}$ \\
\hline \multirow{5}{*}{ Metodología } & Video foro & $\begin{array}{l}\text { "Residuos electrónicos, cam- } \\
\text { bio ambiental" } \\
\text { Resolución de preguntas y/o } \\
\text { situaciones problema } \\
\text { ¿Cuál es la responsabilidad } \\
\text { que se debe asumir para mi- } \\
\text { nimizar el impacto ambien- } \\
\text { tal generado por el desarro- } \\
\text { llo tecnológico? }\end{array}$ \\
\hline & Trabajo individual & $\begin{array}{l}\text { ¿Cómo se relaciona la obso- } \\
\text { lescencia con la basura elec- } \\
\text { trónica? } \\
\text { ¿Por qué las baterías son } \\
\text { contaminantes? } \\
\text { Lectura de imágenes para } \\
\text { identificar la minería urbana }\end{array}$ \\
\hline & $\begin{array}{l}\text { Socialización de } \\
\text { resultados }\end{array}$ & $\begin{array}{l}\text { Diálogo entre los participan- } \\
\text { tes frente al trabajo indivi- } \\
\text { dual, reforzando conceptos } \\
\text { claves. }\end{array}$ \\
\hline & Recomendaciones & $\begin{array}{l}\text { Análisis de las situaciones } \\
\text { planteadas que } \\
\text { permitan una reflexión pro- } \\
\text { funda. }\end{array}$ \\
\hline & Relatoría & $\begin{array}{l}\text { Establecimiento de grupos } \\
\text { de trabajo, quienes realizan } \\
\text { la descripción del ejercicio } \\
\text { desarrollado, la temática } \\
\text { tratada y los compromisos } \\
\text { derivados del taller. Se lee al } \\
\text { iniciar cada taller, a partir del } \\
\text { número } 2 \text {, con el objetivo de } \\
\text { establecer relaciones de ma- } \\
\text { nera continua. }\end{array}$ \\
\hline
\end{tabular}

2. Me comprometo con la adecuada disposición de las baterías de equipos tecnológicos

Identificar los efectos causados por las baterías como residuos tecnológicos en el medio ambiente

\begin{tabular}{l}
\multicolumn{1}{c}{$\begin{array}{c}\text { Metales } \\
\text { Incineración } \\
\text { Rellenos sanitarios }\end{array}$} \\
"Basura tecnológica: La intoxicación \\
silenciosa" “Ecocomputo" \\
Resolución de preguntas y/o situacio- \\
nes problema \\
Lectura de imágenes para describir los \\
efectos que causan las baterías.
\end{tabular}

Esquema de las consecuencias del consumismo en la acumulación de basura tecnológica.

Trabajo individual ¿De qué forma la minería tradicional causa graves efectos en el medio ambiente?

Socialización de resultados

Diálogo entre los participantes frente al trabajo individual, reforzando conceptos claves.

Recomendaciones

Análisis de las situaciones planteadas que permitan una reflexión profunda.

Establecimiento de grupos de trabajo, quienes realizan la descripción del ejercicio desarrollado, la temática tratada y Relatoría los compromisos derivados del taller. Se lee al iniciar cada taller, a partir del número 2, con el objetivo de establecer relaciones de manera continua. 


\section{Taller \\ 1. Desarrollo tecnológico, generación y disposición de la basura electrónica}

Se reconoce que la responsabilidad frente al desarrollo tecnológico es compromiso de consumidores, productores y Gobierno.

Se adquieren conocimientos sobre los diferentes tipos de obsolescencias, las cuales representan las principales causas en la acumulación y crecimiento de la basura tecnológica. Las baterías se iden-

Evaluación tifican como principales contaminantes, debido a los metales que hacen parte de su composición, y al disponerse de manera inadecuada, causan efectos en el medio ambiente.

Se toma la minería urbana como la principal solución para modificar hábitos, y así contribuir en la minimización del impacto ambiental, ya que permite la extracción de metales a través del reciclaje de los RAEE, para elaborar nuevos equipos tecnológicos.
2. Me comprometo con la adecuada disposición de las baterías de equipos tecnológicos

Los estudiantes aceptan que la inadecuada disposición de las baterías afecta recursos como: el agua; contaminándola, el suelo; causando infertilidad, e incluso también afecta la salud de las personas provocando vómitos, diarrea, daño en los riñones, pulmones y producir cáncer.

Se conoce que el consumismo tecnológico se produce principalmente por la obsolescencia inducida, donde los consumidores tienden a cambiar de equipos por su continua oferta y porque desean estar a la vanguardia de la tecnología.

Los estudiantes se preocupan por el impacto ambiental que genera la minería tradicional, ya que permite la extracción de metales, que afectan los recursos naturales, causando niveles altos de contaminación y con ello la pérdida de seres vivos que se encuentran asociados al ecosistema. De esta manera se pretende corregir los hábitos, donde estos apunten hacia una minería sostenible.

\section{Taller}

Propósito

Conceptos clave • Reúso

- Reciclaje

\section{Aplico lo aprendido sobre baterías de} equipos tecnológicos

Desarrollar actividades que comprometen a los miembros de la comunidad educativa con la adecuada disposición de las baterías de equipos tecnológicos teniendo en cuenta sus características.

- Centros de recolección

Resolución de preguntas y/o situaciones problema

Diseño del punto de recolección de baterías.

Trabajo individual Creación del lema del centro de recolección. Elección de la fecha del reciclaje tecnológico.

Metodología

Socialización de resultados

Exposición de los centros de recolección y lemas planteados para seleccionar y realizar mejoras.

Incluir las instrucciones para

Recomendaciones la disposición de los residuos tecnológicos.

\section{Asumo un comportamiento responsable}

Asumir responsablemente el compromiso relacionado con la adecuada disposición de baterías de equipos tecnológicos, como una forma de contribuir con la disminución de la contaminación en el medio ambiente.

- Responsabilidad ambiental

- Compromiso ambiental

Video foro

Trabajo individual

Socialización de resultados

Recomendaciones
“iLlamado de atención! Consumismo obsolescencia programada"

"Programa para recolectar residuos peligrosos"

Resolución de preguntas y/o situaciones problema

Frase dirigida a los habitantes del planeta para crear conciencia acerca de los residuos tecnológicos.

Elaboración de una historieta que plantee una solución en cuanto a la inadecuada disposición de las baterías.

Diálogo entre los participantes frente al trabajo individual, reforzando conceptos claves.

Análisis de las situaciones planteadas que permitan una reflexión profunda. 


3. Aplico lo aprendido sobre baterías de
equipos tecnológicos
Establecimiento de grupos
de trabajo, quienes realizan
la descripción del ejercicio
desarrollado, la temática
tratada y los compromisos
derivados del taller. Se lee al
iniciar cada taller, a partir del
némero 2, con el objetivo de
establecer relaciones de ma-
nera continua.

Se revisa los conocimientos adquiridos y se ponen en práctica, para el diseño del centro de recolección, donde se tiene en cuenta las instrucciones para una adecuada disposición, un logo y un lema.

Los estudiantes reflexionan acerca de la importancia de promover el reciclaje tecnológico, mediante el planteamiento de lemas alusivos a la contaminación tecnológica, cuidado y responsabilidad de la humanidad frente al cuidado del planeta, asumiendo una responsabilidad con el reciclaje tecnológico.

Se interesan en la promoción de la campaña del reciclaje tecnológico, poniendo en práctica los Conocimientos adquiridos, donde se decidió que la opción adecuada para la recolección de baterías sería durante el mes de abril.
4. Asumo un comportamiento responsable

Establecimiento de grupos de trabajo, quienes realizan la descripción del ejercicio desarrollado, la temática tratada y los compromisos derivados del taller. Se lee al iniciar cada taller, a partir del número 2 , con el objetivo de establecer relaciones de manera continua.

La frase demuestra preocupación sobre los efectos de las baterías, y el consumismo. Se resalta la actitud ambiental que se debe asumir frente a la responsabilidad como consumidores, en el reciclaje tecnológico y compromiso frente al cuidado del planeta.

Se plantea las soluciones para la adecuada disposición de baterías, que permite la modificación de hábitos, donde se utilizan los centros de recolección existentes.

Se da importancia a la información, que es preciso adquirir frente al reciclaje tecnológico, la composición de las baterías, la aplicación de la minería urbana, responsabilidad de las empresas, minimización del consumismo tecnológico el cual se asocia con las obsolescencias, para la modificación de los hábitos.

Fuente: Elaboración de la autora.

De acuerdo con la falta de conocimiento, actitudes y hábitos que permiten una disposición adecuada que deben recibir las baterías de equipos tecnológicos, como principales residuos que contienen mayor cantidad de componentes tóxicos, la cual fue detectada en el diagnóstico, se diseña y aplica la Estrategia de Educación Ambiental que se sustenta en la elaboración de cuatro talleres que abarcan los temas relacionados con: el desarrollo tecnológico y disposición de los residuos, impacto ambiental de las baterías, conocimientos prácticos sobre las baterías y análisis sobre el comportamiento ambiental asumido frente a la disposición de residuos tecnológicos.

Mediante los talleres se logra compartir conocimiento acerca de la importancia de reciclar las baterías de equipos tecnológicos, donde los estudiantes cuentan con espacios que permiten observar, dialogar, analizar y reflexionar a partir de diferentes situaciones que se proyectan por medio de videos, imágenes y frases, que permiten el acercamiento con una problemática ambiental que carece de información y compromiso.

A la vez, los espacios que establecen los talleres, promueven la motivación orientada hacia la acción, que hace énfasis sobre la importancia de adquirir una cultura del reciclaje tecnológico, lo que permite la adopción de una actitud comprometida, acorde con un pensamiento racional y consiente frente al cuidado de los recursos, donde se parte del orden y categorización del mundo de manera coherente, satisfaciendo la necesidad de tener una imagen clara y significativa de la problemática que surge al- 
rededor del inadecuado manejo de las baterías como residuos tecnológicos. De manera que las actitudes ayudan al estudiante a ordenar, entender y asimilar la información que puede resultar compleja e impredecible. Además, permite el logro de objetivos deseados, los cuales se relacionan con la adopción de hábitos que fortalecen el cuidado del medio ambiente.

De acuerdo con los resultados obtenidos en cada uno de los talleres, se puede apreciar en primera instancia, la responsabilidad frente al desarrollo tecnológico es compromiso de todos, tanto del gobierno, como productores y consumidores, teniendo en cuenta el principio político llamado: Responsabilidad Extendida del Productor (REP), que propone la reducción del impacto ambiental por iniciativa de los fabricantes, para mejorar la retoma, el reciclaje, la disposición final de los residuos, ofreciendo a los consumidores la posibilidad de disponer estos desechos de forma adecuada. Bajo esta perspectiva, se reconoce la necesidad de adoptar una responsabilidad tecnológica que compete a todos, encaminada hacia la disposición adecuada de los residuos que garantice su reciclaje.

A la vez se reflexiona sobre las consecuencias de los tipos de obsolescencia, las cuales son un gran problema en la acumulación de la basura tecnológica, debido a una falta de compromiso entre fabricantes y consumidores, de manera que la obsolescencia se percibe como el deseo del consumidor de poseer algo, un poco más nuevo y mejor. Ya no se trata de obligar al consumidor a comprar un producto, sino de seducirlo para que lo compre. De manera que la estrategia permite al estudiante reconocer el valor de uso que tienen los equipos tecnológicos y la responsabilidad que se debe adquirir ante la continua oferta de productos.

De acuerdo con la problemática que surge alrededor de la inadecuada disposición de las baterías que requiere la adquisición de conocimientos para el desarrollo de actitudes y há- bitos para asegurar un correcto manejo, se visualiza en la minería urbana los beneficios que permiten favorecer la importancia de disponer de manera adecuada este tipo de residuos tecnológicos, con el propósito de disminuir la explotación de los recursos naturales, ya que tiene un doble impacto positivo; primero, permite recuperar metales o materiales que son cada vez más escasos y cuya obtención, a través de la minería, genera un alto impacto ambiental y social, y segundo, al mismo tiempo frena el impacto que estos residuos generan en el ambiente al degradarse en basurales o rellenos, contaminando el agua, suelo y aire.

Para promover la disposición adecuada de las baterías, se diseñaron centros de recolección en cada institución educativa, los cuales cuentan con instrucciones para que se pueda depositar únicamente los elementos que el contenedor indica. De manera que estas señalan la recolección de baterías de teléfonos celulares, tabletas y computadores portátiles, donde además se resalta la entrega del equipo, en dado caso que la batería se encuentre incorporada. A la vez cuenta con un logo y el lema: "Recicla tu batería y comunícate con el planeta", que se ilustra en la figura 1.

Figura 1. Logo del centro de recolección de baterías de equipos tecnológicos

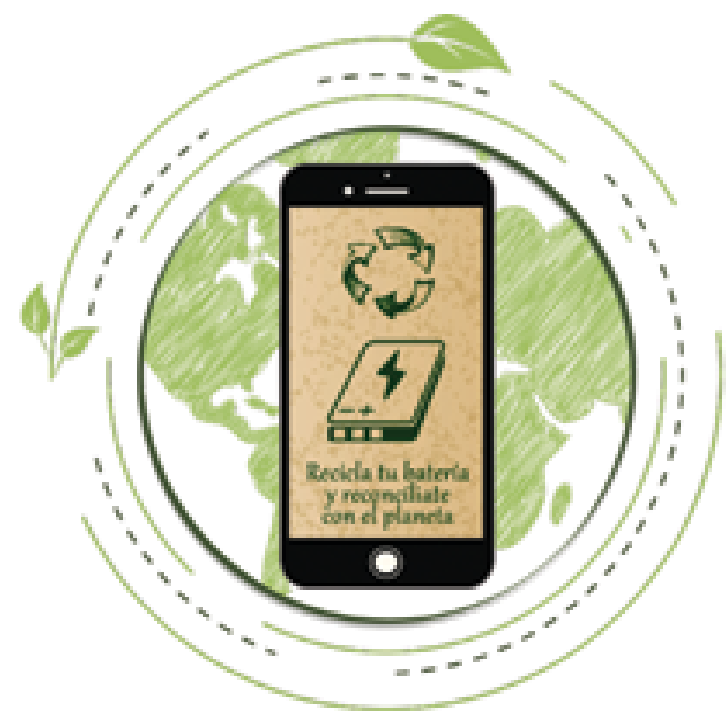

Fuente: Proyecto de Investigación. Las baterías como residuos tecnológicos contaminantes. Departamento de Boyacá. 
De manera que los estudiantes tienen en cuenta que las baterías son componentes con gran potencial de contaminación en los equipos tecnológicos. Estos contaminantes se concentran inadvertidamente y sin control en los rellenos sanitarios y basurales, con consecuencias muy graves y escasamente documentadas. Es así como una vez finalizada la vida útil de las baterías, las cuales no son residuos inocuos, se reconoce la necesidad de ser tratadas y recicladas mediante la posibilidad que se abre al disponerlas en los puntos de recolección, que además permiten ser separadas del flujo de los residuos domiciliarios comunes y dispuestos de manera segura. Así que se contempla en las acciones relacionadas con el desecho de estos residuos en la basura y la acumulación en hogares, como soluciones inseguras y ambientalmente irresponsables.

Es así como los talleres permitieron el planteamiento de soluciones y compromisos institucionales, en la promoción de una cultura ambiental ante la necesidad del reciclaje tecnológico, que hace posible la recolección de baterías de teléfonos celulares, tabletas y computadores portátiles en cada institución educativa, en la tabla 4 se ilustra la cantidad de baterías recolectadas durante el mes de abril.

Tabla 4. Cantidad de baterías recolectadas

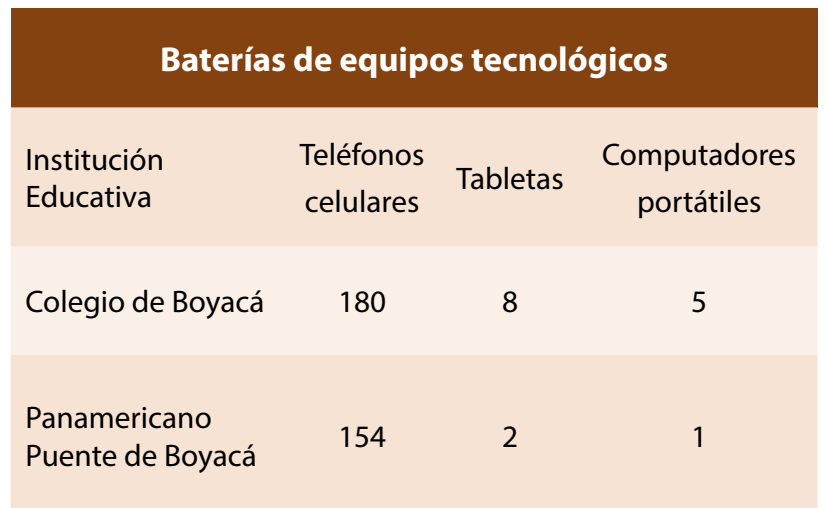

Fuente: Elaboración de la autora.
El Colegio de Boyacá es una institución que lleva a cabo diferentes proyectos pedagógicos, ve la necesidad de vincular los estudiantes en la solución de problemas ambientales y decide dar continuidad a la Estrategia de Educación Ambiental propuesta con la participación de los estudiantes de servicio social, quienes extienden la invitación a los otros estudiantes, con el apoyo de los docentes de Ciencias Naturales; para de esta manera contribuir con el establecimiento de una cultura del reciclaje tecnológico.

De igual manera, en la Institución Educativa Panamericano Puente de Boyacá se garantizan acciones para la sostenibilidad de la estrategia, por medio de su inclusión a través del plan de área de Ciencias Naturales, donde los docentes establecen un compromiso que permita fomentar en el estudiante el espíritu investigativo, y se despierte el interés y la curiosidad para la resolución de problemas ambientales mediante el desarrollo de la Estrategia de Educación Ambiental.

\section{Evaluación de la Estrategia de Educación Ambiental}

La Estrategia de Educación Ambiental para la disposición de baterías de equipos tecnológicos se evalúa mediante una entrevista semiestructurada que se realiza al $20 \%$ de la muestra seleccionada, teniendo en cuenta tanto los grupos focales como el grado noveno de cada institución educativa.

La evaluación de la estrategia se realiza mediante el programa ATLAS.ti versión 7 y los resultados obtenidos permiten identificar situaciones que evidencian logros alcanzados en cada una de las categorías de análisis resultantes del diagnóstico, como son: la adquisición de conocimientos, desarrollo de actitudes y hábitos, los cuales se ilustran en la tabla 5. 
Tabla 5. Evaluación de la Estrategia en Educación Ambiental

\begin{tabular}{|c|c|}
\hline Categoría de análisis & Situación \\
\hline Conocimiento & $\begin{array}{l}\text { Reconoce la composición de las baterías. } \\
\text { Conoce las consecuencias del consumismo tecnológico. } \\
\text { Interpreta la necesidad de desarrollar la minería urbana, como prin- } \\
\text { cipal solución para minimizar el impacto ambiental que origina la } \\
\text { minería tradicional en el uso desmedido de los recursos. } \\
\text { Identifica los diferentes tipos de obsolescencias que promueven el } \\
\text { consumismo y acumulación de residuos tecnológicos. } \\
\text { Se conoce la falta de responsabilidad ambiental que se debe adqui- } \\
\text { rir frente a la disposición adecuada de las baterías, donde se haga } \\
\text { uso de los centros de recolección. }\end{array}$ \\
\hline Actitud & $\begin{array}{l}\text { Asumen una responsabilidad frente a las baterías como residuos } \\
\text { tecnológicos contaminantes. } \\
\text { Los estudiantes se preocupan por realizar una adecuada disposi- } \\
\text { ción, haciendo uso de los centros de recolección para promover los } \\
\text { beneficios del reciclaje. } \\
\text { Reflexiona acerca de los efectos que ocasionan las baterías en el me- } \\
\text { dio ambiente. } \\
\text { Se preocupa por el impacto ambiental que generan las baterías } \\
\text { como residuos tecnológicos, donde la mayoría de las personas no } \\
\text { lo percibe. } \\
\text { Se interesa por el planteamiento de soluciones frente al desarrollo } \\
\text { tecnológico. } \\
\text { Se acepta la responsabilidad que debe adquirir tanto productores } \\
\text { como consumidores en cuanto a los residuos que se producen. } \\
\text { Se dan cuenta de la importancia de proyectar la minería urbana } \\
\text { como la principal solución sostenible para la protección de los re- } \\
\text { cursos naturales. }\end{array}$ \\
\hline Hábito & $\begin{array}{l}\text { Los estudiantes hacen uso de los centros de recolección para la dis- } \\
\text { posición de baterías. } \\
\text { Se corrigen acciones en cuanto al desecho de estos residuos en los } \\
\text { rellenos sanitarios y en el hogar. } \\
\text { Interpreta la importancia de hacer uso de los centros de recolección, } \\
\text { con el propósito de minimizar el impacto ambiental y avanzar hacia } \\
\text { un desarrollo sostenible }\end{array}$ \\
\hline
\end{tabular}

Fuente: Elaboración de la autora.

Las respuestas dadas por los estudiantes evidencian las ventajas que ofrece la estrategia en la construcción de conocimientos ambientales, acerca de la importancia de realizar una adecuada disposición de las baterías para garantizar el reciclaje tecnológico y prevenir el impacto ambiental, en la que se promueven los beneficios de la minería urbana y se evita el consumismo tecnológico.

Mediante los espacios de reflexión que brinda la estrategia, los estudiantes modifican su forma de pensar a partir de los conocimientos adquiridos que permiten comprender la necesidad de utilizar los puntos de recolección para garantizar una adecuada disposición y, por ende, el apro- vechamiento de la materia prima que hace parte de la composición de las baterías.

De acuerdo con los resultados descritos anteriormente, la Estrategia de Educación Ambiental fomenta conocimientos, hábitos y actitudes para una adecuada disposición de las baterías como residuos tecnológicos contaminantes, ya que al representar un plan de acción ante una tarea que requiere una actividad cognitiva que implica aprendizaje, resalta el carácter procedimental que tiene todo aprendizaje. Es así como permite elegir, coordinar y aplicar las habilidades, vinculándolas con el aprendizaje significativo y con el aprender a aprender (Monereo, citado por González y Díaz, 2006, p. 9). 
Adoptar una Estrategia de Educación Ambiental para la adecuada disposición de las baterías de equipos tecnológicos contribuye a la generación de cambios y compromisos en la sostenibilidad del medio ambiente. Todo gira alrededor de la idea de progreso, en especial la tecnología, donde este progreso se mueve entre dos infinitos: el infinito de los recursos de la Tierra y el infinito del futuro. Se pensaba que la tierra era inagotable en sus recursos y que podíamos avanzar indefinidamente en la dirección del futuro. La conciencia de crisis reconoce: que los recursos tienen límites, ya que no todos son renovables; que el crecimiento indefinido hacia el futuro es imposible, porque no se puede universalizar el modelo de crecimiento para todos y para siempre (Boff, 2002, p. 25).

\section{Divulgación de la Estrategia de Educación Ambiental}

La divulgación de la Estrategia de Educación Ambiental orientada al manejo responsable de los residuos tecnológicos más frecuentes se divulgó mediante la Emisora del Colegio de Boyacá, 102.6 FM, donde los estudiantes de servicio social participan activamente en la elaboración y grabación de los diferentes programas de radio, que abordan las temáticas claves sobre la importancia de realizar una adecuada disposición de las baterías de equipos tecnológicos. Es así como, a través de este medio se informa, motiva e invita, tanto a las demás sedes que pertenecen a cada institución como a la región, a hacer parte de esta campaña ambiental, con frases alusivas al reciclaje tecnológico y conceptos de interés, teniendo en cuenta que la emisora tiene una amplia cobertura en la ciudad de Tunja y municipios cercanos.

De tal forma que se comparten ocho programas de radio que abordan las temáticas que fundamentan la Estrategia de Educación Ambiental que se ilustran en la tabla 6 .

\section{Tabla 6. Descripción de los programas de radio}

Programa de radio

Educación Ambiental para

la disposición de baterías de equipos tecnológicos

Características del avance tecnológico

RAEE

Características de las baterías de equipos tecnológicos

Efectos de las baterías de equipos tecnológicos

Minería urbana

\section{Fundamento}

Este primer programa busca, presentar el objetivo de la estrategia de Educación Ambiental desarrollada en las dos instituciones educativas del departamento de Boyacá, y comunicar de manera general los temas que se tratarán en los diferentes programas.

Se aborda la evolución constante que han tenido los equipos tecnológicos como: teléfonos celulares, tabletas y computadores portátiles, donde se tiene en cuenta la falta de responsabilidad frente a los residuos que este tipo de tecnología produce, debido al continuo cambio de equipos.

A través de este programa se explica el significado de los RAEE, y se da a conocer el tipo de equipos que hacen parte de este grupo de residuos. Además, se da a conocer las características de estos y sus implicaciones en el medio ambiente

Se da a conocer los componentes tóxicos de las baterías que las convierten en los principales residuos contaminantes, y su clasificación de acuerdo con su composición química. De igual forma se explica la importancia de reciclar las baterías teniendo en cuenta su contenido en metales.

Se expresa la manera cómo las baterías de equipos tecnológicos se convierten en principales residuos contaminantes, que afectan los recursos naturales debido a su composición.

Expone la principal solución para minimizar el impacto ambiental que produce la minería tradicional, donde se aproveche los metales de las baterías en desuso, como materia prima para la elaboración de nuevos equipos. 


\begin{tabular}{|c|c|}
\hline Programa de radio & Fundamento \\
\hline $\begin{array}{l}\text { Disposición de baterías de } \\
\text { equipos tecnológicos }\end{array}$ & $\begin{array}{l}\text { Especifica la importancia de los centros de recolección para la ade- } \\
\text { cuada disposición de residuos tecnológicos, y se da a conocer los } \\
\text { puntos certificados que existen en Colombia y el departamento, } \\
\text { donde se incluyen los sitios de recolección que están dispuestos en } \\
\text { las dos instituciones educativas y sus respectivas sedes y se extiende } \\
\text { la invitación para que los oyentes hagan parte de esta campaña. }\end{array}$ \\
\hline $\begin{array}{c}\text { Compromiso como persona y } \\
\text { como sociedad }\end{array}$ & $\begin{array}{l}\text { Se comparten reflexiones acerca de la importancia de adoptar actitu- } \\
\text { des y hábitos en torno al reciclaje de las baterías de equipos tecnoló- } \\
\text { gicos, donde se conciba en la minería urbana las principales ventajas } \\
\text { que trae el reciclaje tecnológico. }\end{array}$ \\
\hline
\end{tabular}

Fuente: Elaboración de la autora.

\section{Conclusiones}

El diagnóstico para el desarrollo de la estrategia permite identificar la falta de conocimiento acerca de lo que se debe hacer con las baterías como residuos tecnológicos al final de su vida útil, lo que provoca que estas sean dispuestas en la basura o almacenadas en el hogar, sin percibir los efectos que ocasionan en el medio ambiente.

La Estrategia de Educación Ambiental, entendida como un plan de acción ante una tarea que requiere una actividad cognitiva que implica aprendizaje, permite promover conocimientos para modificar actitudes y hábitos en torno a una adecuada disposición de las baterías de equipos tecnológicos, ya que representan un foco de contaminación debido a los metales que hacen parte de su composición.

El diseño metodológico que orienta la Estrategia de Educación Ambiental se basa en la investigación acción, este involucra la transformación y mejora de la realidad, con base en el desarrollo de talleres que permiten la reflexión y el compromiso que se asumen frente a los residuos tecnológicos, para asegurar una adecuada disposición y evitar a la vez el desarrollo de las obsolescencias. Para el logro de este objetivo, encaminado hacia una adecuada disposición, se requiere la adquisición de conocimientos para el desarrollo de hábitos y actitudes en el estudiante que le permita comprender la importancia y necesidad de hacer uso de los centros de recolección, para asumir una responsabilidad frente a las baterías como residuos tecnológicos contaminantes y corregir acciones en cuanto al desecho de estos residuos.

El análisis de los datos cualitativos mediante el software ATLAS.ti versión 7 permite la codificación para organizar, reagrupar y gestionar las respuestas dadas por los estudiantes, de manera creativa y, al mismo tiempo sistemática donde se potencializa la capacidad y calidad del análisis.

Los talleres constituyen la base de la Estrategia en Educación Ambiental, y por medio de estos se promueve el conocimiento para el desarrollo de hábitos y actitudes mediante el acercamiento del estudiante a las ventajas que tiene el reciclaje de baterías para permitir visualizar la fuente de recursos que estas significan, debido a que provee materia prima que sirve para la elaboración de equipos tecnológicos nuevos. Es así que por medio de los espacios que brindan los talleres se contribuye al reciclaje tecnológico, a partir del uso de los puntos de recolección que permiten asegurar un manejo adecuado de las baterías, a fin de prevenir los efectos que pueden causar en el medio ambiente. 


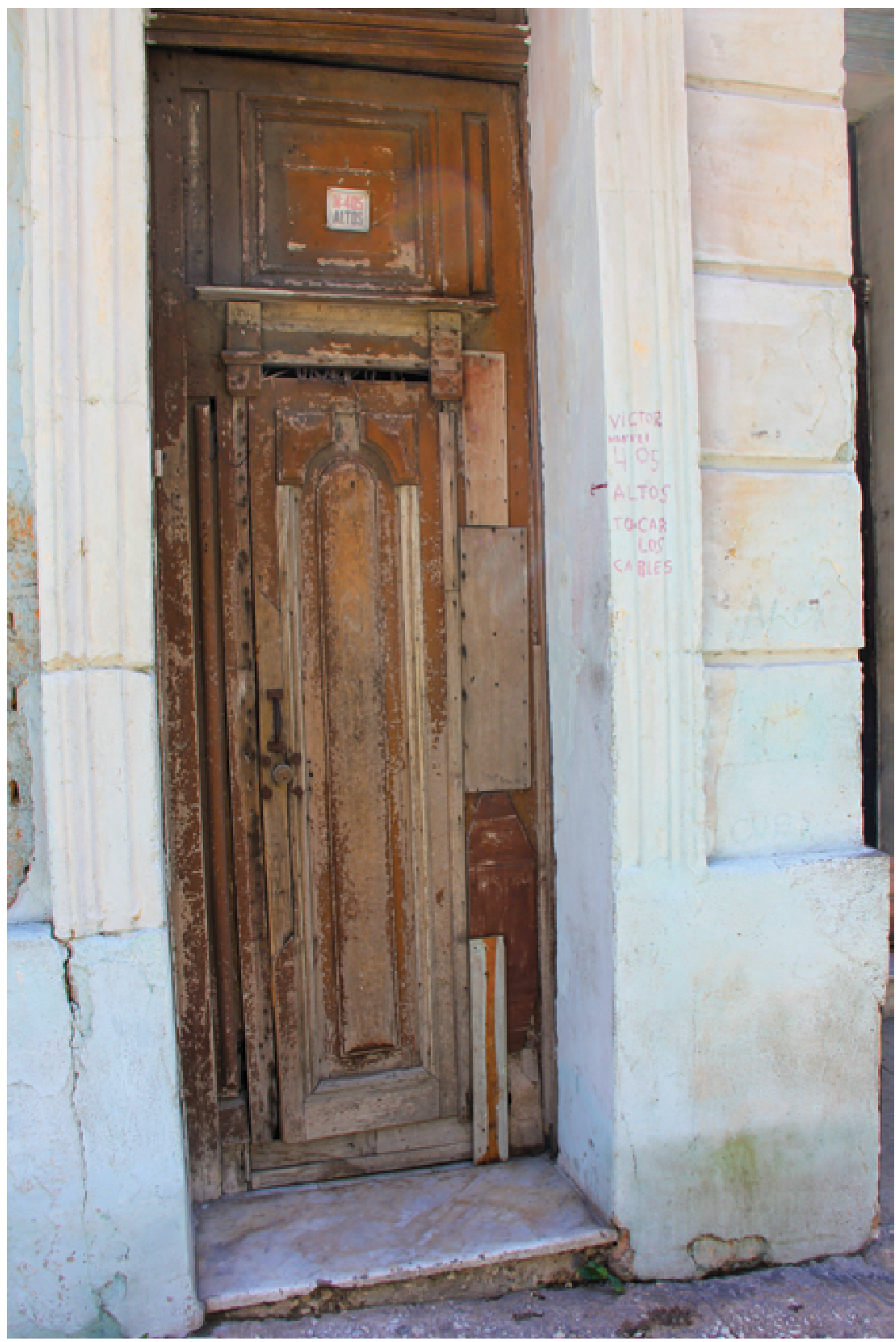


La implementación de la Estrategia de Educación Ambiental permite la adquisición de conocimientos para la comprensión de la problemática ocasionada por los efectos que provoca la inadecuada disposición de las baterías de equipos tecnológicos, y el desarrollo de actitudes y hábitos relacionados con el manejo responsable de este tipo de residuos, teniendo en cuenta el papel de la ética ambiental para tratar desde un punto racional los problemas relacionados con el medio ambiente, pues la capacidad de intervención sobre el medio es posible si se fortalece mediante una base racional para tomar decisiones ambientalmente correctas.

El uso de la radio para la divulgación de la estrategia permite compartir información acerca de la importancia de asegurar una adecuada disposición y tratamiento de las baterías. A la vez motiva e invita a la comunidad a hacer parte de la solución de esta problemática, donde se adopten hábitos que garanticen la sostenibilidad del planeta.

Al promover puntos de recolección para la disposición de baterías, se permite la modificación de hábitos que benefician el aprovechamiento de la materia prima contenida en las baterías, donde se garantiza un manejo ambientalmente adecuado. Además de proyectar una responsabilidad ambiental frente a los residuos que la tecnología produce, a fin de prevenir las consecuencias que provocaría una inadecuada disposición.

Los principios orientadores del PEl que favorecen la estrategia en cada institución educativa son: Colegio de Boyacá: la capacidad de aprender y mejorar el mundo y la formación en competencias, donde la estrategia se fortalece a través del proyecto transversal que se lleva a cabo. Por otro lado, en la Institución Educativa Panamericano Puente de Boyacá los principios que fortalecen la estrategia son: el trabajo en equipo por la conservación del medio ambien- te y el uso consciente de la tecnología, donde esta se acoge bajo el Proyecto Transversal de Educación Ambiental.

Las dos instituciones participantes asumen el compromiso de contribuir con la cultura del reciclaje tecnológico, la Institución Educativa Panamericano Puente de Boyacá lo hace desde la adopción de la estrategia, mediante su inclusión en el plan del Área de Ciencias Naturales y el Colegio de Boyacá, vincula la estrategia como proyecto ambiental que requiere la participación y apoyo de los estudiantes de servicio social y docentes de Ciencias Naturales.

La promoción y adopción de esta estrategia es necesaria para que la sociedad se eduque a nivel de conocimientos y comportamientos ambientalmente responsables y sostenibles con el medio ambiente, que permita minimizar el impacto ambiental que genera la inadecuada disposición de las baterías de equipos tecnológicos, debido a que provoca una creciente destrucción de los recursos naturales. Es así como se debe contemplar en la basura tecnológica, la principal fuente de recursos capaz de proveer la materia prima necesaria para el continuo desarrollo tecnológico.

\section{Referencias}

Avendaño, W. (2013). Un modelo pedagógico para la educación ambiental desde la perspectiva de la modificabilidad estructural cognitiva. Recuperado de http:// www.scielo.org.co/scielo.php?script=sci_arttext\&pid $=$ S1909-24742013000100009

Boff, L. (2002). El cuidado esencial. Ética de lo humano, compasión por lo humano. Recuperado de https://www. revistautopia.org/leonardo-boff-el-cuidado-esencialetica-de-lo-humano-compasion-por-la-tierra/

González, D., y Díaz, Y. (2006). La importancia de promover en el aula estrategias de aprendizaje para elevar el nivel académico en los estudiantes de psicología. Revista lberoamericana de Educación, 40(1), 1-17. Recuperado de file://C:/1379Gonzalez.pdf

Mora, F., Quitiaquez, L., y Rengifo, B. (2012). La educación ambiental una estrategia pedagógica que contribuye a la solución de la problemática ambiental en Colombia. Recuperado de http://www.ub.edu/geocrit/coloquio2012/ actas/06-B-Rengifo.pdf 